\title{
Higher Sensitivity and Earlier Identification of Celiac Disease Autoimmunity by a Nonradioactive Assay for Transglutaminase Autoantibodies
}

\author{
Zhiyuan Zhao, ${ }^{1,2}$ Dongmei Miao, ${ }^{2}$ Kathleen Waugh, ${ }^{2}$ Iman Taki, ${ }^{2}$ Fran Dong, ${ }^{2}$ Edwin Liu, ${ }^{3}$ \\ Marian Rewers, ${ }^{2}$ Yu Liu, ${ }^{1,4}$ and Liping $Y u^{2}$ \\ ${ }^{1}$ Department of Endocrinology, 2nd Hospital of Jilin University, Changchun, Jilin, China \\ ${ }^{2}$ Barbara Davis Center for Childhood Diabetes, University of Colorado Denver, Aurora, CO, USA \\ ${ }^{3}$ University of Colorado Children's Hospital, Aurora, CO, USA \\ ${ }^{4}$ Sir Run Run Hospital, Nanjing Medical University, Nanjing, China \\ Correspondence should be addressed to Yu Liu; liuyu73@hotmail.com and Liping Yu; liping.yu@ucdenver.edu
}

Received 19 September 2016; Accepted 6 December 2016

Academic Editor: Isabel Comino

Copyright (C) 2016 Zhiyuan Zhao et al. This is an open access article distributed under the Creative Commons Attribution License, which permits unrestricted use, distribution, and reproduction in any medium, provided the original work is properly cited.

\begin{abstract}
Higher sensitive transglutaminase autoantibody (TGA) assay will detect the onset of celiac disease (CD) autoimmunity earlier. In developing a nonradioactive assay for TGA, we utilized electrochemiluminescence (ECL) technology and compared it to a highperformance radioimmunoassay (RIA) currently being used to screen patients with type 1 diabetes (T1D) and genetically at-risk individuals for CD. We selected 183 T1D patients with 60 patients having received biopsy and analyzed 396 sequential samples from 73 young children longitudinally followed up with TGA seroconversion, with 27 undergoing biopsy. In addition, 112 age-matched healthy control subjects were included in the study. With the 99th percentile of specificity, the ECL assay detected significantly more TGA positivity among patients with T1D (133/183) than RIA (114/183) and more of the sequential samples (34\%) from 73 children than RIA (18\%). The TGA assay performed by ECL was positive in all 59 subjects with villous atrophy. Among 73 longitudinally followed up children, ECL assay had earlier detection of TGA on 34 children by a mean of 2.5 years. In conclusion, the new TGA assay by ECL has a higher sensitivity than the current RIA assay and may better predict the onset of CD.
\end{abstract}

\section{Introduction}

Celiac disease (CD) is well defined as a chronic small intestinal autoimmune enteropathy precipitated by exposure to dietary gluten in genetically predisposed individuals. The prevalence of $\mathrm{CD}$ is very high with screening studies suggesting an increasing frequency from $0.3 \%$ up to $3 \%[1-6]$. The clinical presentation of $\mathrm{CD}$ is highly variable and targeted screening based on clinical symptoms is not effective in diagnosing the majority of individuals with $\mathrm{CD}$ in the general population [7]. Therefore, many individuals remain undiagnosed.

Determination of autoantibodies to tissue transglutaminase (TGA) is currently the most effective single serologic test for the identification of $\mathrm{CD}$ or the development of autoimmunity associated with $\mathrm{CD}$ [8]. Routine screening is recommended for individuals considered to be at high risk, including those with type 1 diabetes [9] and those with a family member with CD among others [10]. Very recently, a successful measurement of TGA in saliva samples was reported [11] that would be a convenient way for large cohort screening, especially among young children. In 2009, a TGA workshop with a large international collaborative effort toward improving and standardizing TGA measurement was organized [12]. The workshop found that radioimmunoassays (RIAs), in general, were more quantitative and more sensitive than standard ELISA assays in detecting low-titer sera (including serially diluted samples). Such assays with higher sensitivity allow detection of the earliest signs of the development of CD autoimmunity, which is critical for such studies investigating environmental triggers of autoimmunity such as 
in the Diabetes Autoimmunity Study in the Young (DAISY) [13] and the large international clinical trial, the Environmental Determinants of Diabetes in the Young (TEDDY) [14]. However, the RIA for TGA is not widely accepted due to the necessity of radioactivity and limited access to laboratories that can perform this assay, as opposed to currently utilized solid-phase assays. We have recently developed and extensively validated nonradioactive islet autoantibody assays for type 1 diabetes using electrochemiluminescence (ECL) detection with excellent sensitivity and specificity compared to the current standard RIAs for islet autoantibody measurements [15-18]. In the present study, we developed a nonradioactive TGA assay with ECL technology using a similar assay format as for islet autoantibody measurement. We analyzed TGA by ECL in 183 selected patients with type 1 diabetes who had the TGA measured by RIA and 396 sequential samples from 73 children who were longitudinally followed up with TGA seroconversion. The comparisons with current standard RIA for assay sensitivity, specificity, and the time of TGA seroconversion were analyzed.

\section{Research Design and Methods}

2.1. Definitions. In this study, CD is defined as having an intestinal biopsy showing a Marsh score of 2 or greater by original Marsh criteria [19]. CD autoimmunity is defined as having persistent TGA positivity on 2 or more sequential measurements done over time.

2.2. Subjects. The serum samples were from 183 patients with type 1 diabetes who were followed up having routinely TGA screened by radioimmunoassay (RIA) at the Barbara Davis Center. The patient ages were ranged from 2.2 to 41.7 years with median age of 13.0 and $51 \%$ were female (94/183). Most patients with TGA positivity were positive on more than one occasion, and 60 patients had an intestinal biopsy. Of these, 42 were diagnosed with CD. In addition, we analyzed 73 subjects from the DAISY birth cohort (having a genetic risk for CD) having a total of 396 serial samples obtained longitudinally over a period of 15 years. TGA positivity was identified in all of them by RIA at some point during the follow-up. Twenty-seven of these subjects were biopsied during their follow-up and 17 were diagnosed with CD based on biopsy findings. Of the 112 gender-matched normal control samples tested, all were negative for TGA by RIA. Signed written informed consents were obtained from participants and the study was approved by the Institutional Review Board of the University of Colorado.

2.3. ECL-TGA Assay. The method of the ECL-TGA assay was adopted from the format of an ECL-GADA assay previously published [17]. The interaction of antibodies in sera with labeled antigen was completed in the liquid phase. One transglutaminase antigen labeled with the biotin allows capture on the streptavidin coated solid phase. The other transglutaminase antigen with the Sulfo-tag provides electrochemical light emission for detection of the captured complex. Both the biotinylated and Sulfo-tagged transglutaminase were used as competitors and tested in our standard
RIA. Both modified molecules were able to compete well with S-35 transglutaminase for binding to TGA in patient sera. Following a series of optimization steps, the ECL assay protocol described below was used for all experiments. In brief, $4 \mu \mathrm{l}$ of serum premixed with $16 \mu \mathrm{l}$ of PBS buffer was incubated with $20 \mu \mathrm{l}$ of antigen buffer containing both Sulfo-tag labeled transglutaminase protein (DIARECT AG, Freiburg, Germany) at the concentration of $100 \mathrm{ng} / \mathrm{ml}$ and biotin-labeled transglutaminase protein at the concentration of $400 \mathrm{ng} / \mathrm{ml}$ in PBS containing 5\% BSA for overnight at $4^{\circ} \mathrm{C}$. On the 2 nd day, $30 \mu$ l of overnight incubates was added per well onto a streptavidin coated plate (MSD, Gaithersburg, $\mathrm{MD}$ ) and incubated for 1 hour at room temperature. After 3 rounds of washing, the plate was counted on an MSD counter, Imager 2400 (MSD, Gaithersburg, MD). The results were expressed as an index against our internal standard positive control serum, the same standard positive control serum used for the RIA. The assay upper limit of normal range (index 0.015 ) was set at $>99$ th percentile of 112 normal control samples that were characterized as TGA negative by RIA. The interassay coefficient variations $(\mathrm{CV})$ were $8.1 \%(n=30)$ with index value around 1.0 and $16.2 \%(n=30)$ with index value around 0.05 .

2.4. RIA-TGA. The method of RIA-TGA was published previously [20] and the upper limit of normal (index 0.050) was established as the 100th percentile of 184 healthy control subjects. The interassay CV for the sample was $8.9 \%(n=250)$ with index value around 1.0 and $19.3 \%(n=15)$ with index value around 0.09 .

2.5. Statistics. Statistical analyses were performed using correlation analysis for TGA levels of two assays, McNemar's test for comparing the sensitivity, with PRISM 4.0 version software (GraphPad Software Inc., San Diego, CA). A twotailed $p$ value with an alpha level for significance was set at 0.05 .

\section{Results}

3.1. Assay Sensitivity Titration. To titrate the sensitivity of ECL-TGA assay and compare it with a current highperformance RIA-TGA assay, 6 TGA positive samples from 6 patients studied, respectively, with confirmed clinical CD by biopsy were in a serial of $1: 1$ dilution with a normal control serum to a maximum of 1:4096 dilution and measured for TGA by both ECL and RIA. The levels of TGA titrated with ECL and RIA were both plotted in Figure 1 with solid line (dark color) for ECL and dotted line (gray color) for RIA, respectively. The last titration points showing TGA positive for RIA versus ECL were $1: 64$ versus $1: 512$ for patient $1,1: 128$ versus $1: 1024$ for patient $2,1: 8$ versus $1: 32$ for patient $3,1: 16$ versus $1: 64$ for patient $4,1: 8$ versus $1: 32$ for patient 5 , and $1: 8$ versus $1: 32$ for patient 6 , which clearly demonstrated that TGA measured by ECL method was much more sensitive than that by RIA.

3.2. Higher Sensitivity of ECL Than RIA in Detecting TGA in Patients with Type 1 Diabetes. With similar assay specificity 


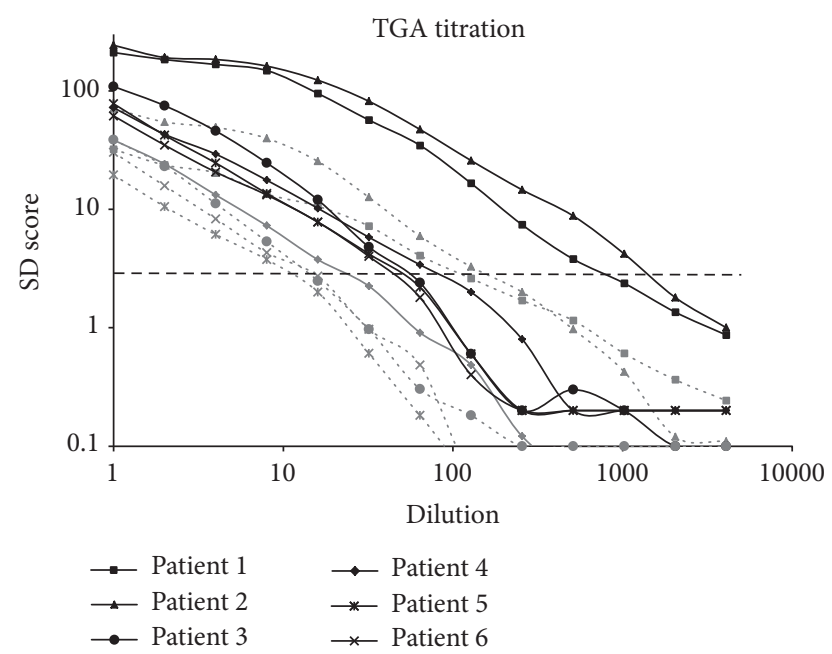

Figure 1: Titration of TGA by both RIA and ECL assay using a TGA positive sample from six of T1D patients studied with confirmed clinical CD by biopsy (marked patient 1 , patient 2 , patient 3 , patient 4 , patient 5 , and patient 6 , respectively). The samples were in a serial of $1: 1$ dilution with a normal control serum to a maximum of 1:4096 dilutions. The solid curves were TGA levels from ECL assay and the dotted curves with gray color were TGA levels from RIA. A horizontal dotted line represents assay cut-offs for both ECL and RIA at 3 standard deviation (SD) scores. The SD scores are calculated with the following formula: (index value - mean index of controls)/SD.

set for both assays among healthy controls, the ECL assay detected significantly more TGA positivity among patients with type 1 diabetes $(73 \%, 133 / 183)$ than RIA $(62 \%, 114 / 183$; $p=0.04)$. The levels of TGA between ECL and RIA as shown in Figure 2 correlated well $\left(R^{2}=0.3418, p<0.0001\right)$. Of the 183 patients, 60 received intestinal biopsy upon their positive TGA results at the time by RIA and 42 were found biopsy positive. Many samples studied from these 60 biopsy patients were months to years after biopsy and the levels of TGA were found gradually declined during the follow-up (data not shown). Four samples in the present study from 3 patients with positive biopsy and one with negative biopsy became TGA negative by RIA (arrow-pointed in Figure 2) and dropped below the assay cut-off while ECL-TGA were still detectable for 3 of these 4 patients, 2 biopsy positive and one biopsy negative, and rest of biopsy patients were all positive for ECL-TGA. Compared between biopsy positive and negative subjects studied, the levels of TGA in mean values had no significant differences with both ECL $(0.77 \pm$ 0.83 versus $0.58 \pm 0.50 ; p=0.57)$ and RIA ( $0.37 \pm 0.35$ versus $0.29 \pm 0.24 ; p=0.79$ ).

3.3. Earlier Detection of TGA by ECL Than RIA in Longitudinally Followed Children. The group of 396 sequential samples from 73 children from DAISY who were longitudinally followed was tested using the ECL assay. These individuals were confirmed to have been TGA positive by RIA at some point in their follow-up. The levels of TGA between ECL and RIA were well correlated $\left(R^{2}=0.5281, p<0.0001\right)$ as shown in Figure 3. TGA was detected in a total of 136 of the 398

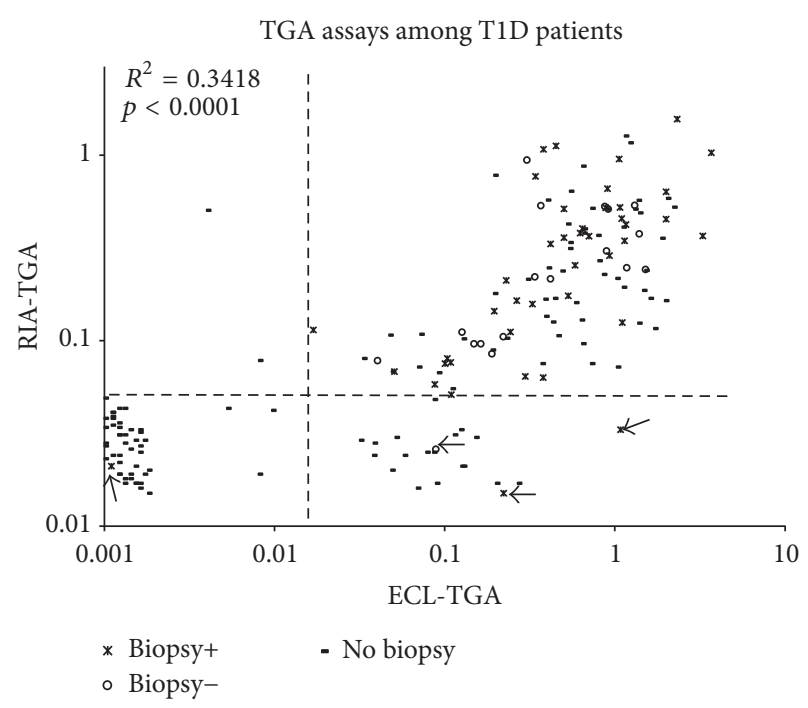

FIGURE 2: TGA levels from ECL assay and our current standard RIA were compared among 183 patients with type 1 diabetes. The cases without or with biopsy+ or - are shown in different markers. Four cases from biopsy group that became TGA negative by RIA were pointed by arrows.

sequential samples by ECL even though 64 of these 136 (47\%) were undetectable by RIA. Among these 73 children with confirmed TGA positive seroconversion during the followup, ECL detected seroconversion earlier in 34 children when compared to RIA, identifying the onset of CD autoimmunity in these children by a mean of 2.5 years ( 0.8 to 13 years) earlier (Figure 4). In those 17 out of 27 with biopsy-confirmed disease, ECL detected TGA earlier than the RIA in 10 of these children by a mean of 2.1 years (range 0.8 to 6 years). The other 7 biopsy positive children detected TGA at the same clinical visit times by both ECL and RIA.

\section{Discussion}

The use of TGA assays with even greater sensitivity is invaluable in longitudinal cohort studies that follow the natural history of the development of $\mathrm{CD}$ autoimmunity. In particular, earlier detection of an immunologic change relevant to CD helps identify potential environmental triggers for the development of autoimmunity by reducing the apparent "lag time" between the two events during analysis. The gold standard for which ECL was compared to the RIA in this study. In children who are undergoing screening for $\mathrm{CD}$ because of an increased genetic risk (such as having type 1 diabetes or a family member with $\mathrm{CD}$ ), autoantibody positivity often precedes the development of intestinal injury. Therefore, highly sensitive assays are utilized in this setting, and in clinical research, to detect the earliest signs of $C D$ autoimmunity, which may lead to CD. It is clearly shown that different organ-specific autoimmune diseases are greatly overlapped. $\mathrm{CD}$ and type 1 diabetes share the high-risk HLA Class II of DR3-DQ2 and DR4-DQ8, and non-HLA genetic susceptibility in Caucasian population and their clinical phenotypes overlap in up to $10 \%$ of the patients [20]. 


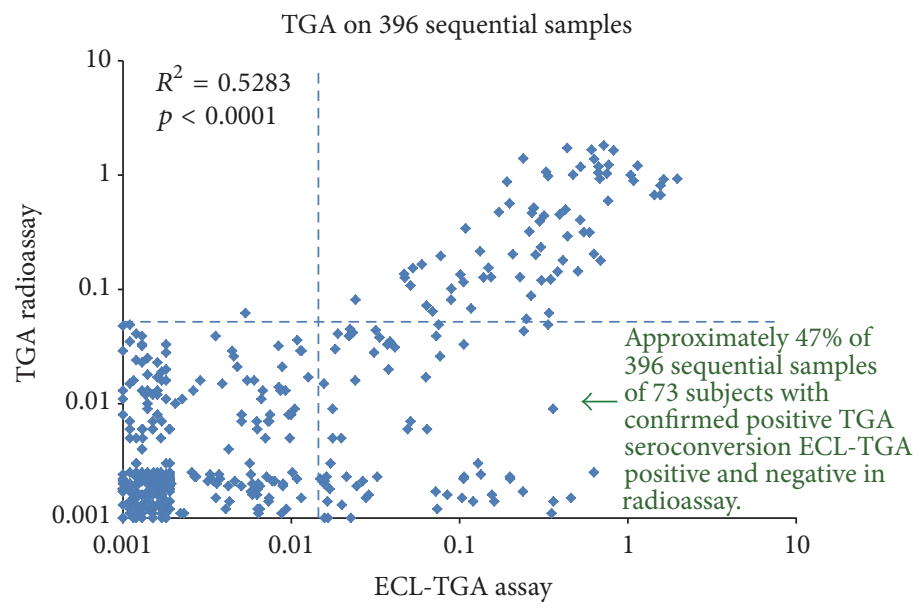

FIGURE 3: TGA levels from ECL assay and our current standard RIA were compared on 396 sequential samples from 73 children who were confirmed TGA positive seroconversion during the longitudinal follow-up.

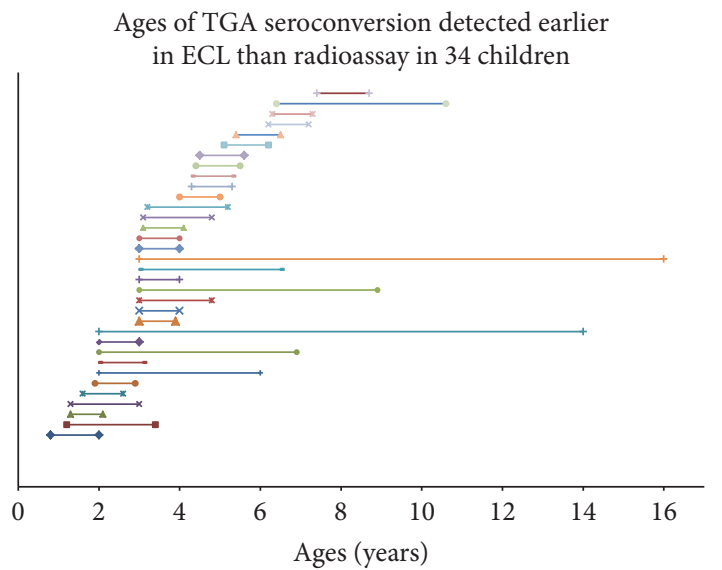

FIGURE 4: TGA were detected earlier in age in 34 children with ECL assay than RIA. The beginning of each line is TGA detecting age with ECL assay for a child and the end of line is TGA detecting age with RIA.

In DAISY and TEDDY, all study participants are screened for CD autoimmunity by testing for TGA at least yearly. Persistent TGA positivity and CD are major endpoints in both studies [21,22].

This TGA assay, performed by ECL, was modified from techniques currently utilized to measure islet autoantibodies. One reason why this assay may be superior to RIA in detecting the presence of TGA is because all immunoglobulin subclasses are able to react with the transglutaminase antigen and therefore could be captured in the ECL assay format (including $\operatorname{Ig} \mathrm{A}, \operatorname{IgG}, \operatorname{Ig} M$, or even $\operatorname{IgE}$ and $\operatorname{IgD}$ ). In comparison, RIA detects only immunoglobulin subclass, IgA. In addition, when measuring islet autoantibodies, the ECL assay detects the more disease-specific high-affinity, high-risk autoantibodies $[15,17,18]$ compared to RIA which detects more low-affinity, low-risk autoantibodies. This feature of disease specificity for TGA will need to be explored in CD.

It has been previously reported that higher TGA levels correlate better with villous atrophy [23]; assays performed by RIA generally have superior quantitative ability when measuring and reporting TGA. In the present group of patients studied, there were no correlations of higher TGA levels with positive intestinal biopsy found for both RIA and new ECL assay. It might be because many samples in the present study were months to years after biopsy and they will not reflect their actual TGA levels at the time of biopsy. Further study will be needed in determining the dynamics of $\mathrm{CD}$ autoimmunity and exploring the disease specificity using this new highly sensitive TGA assay.

\section{Competing Interests}

None of the authors has any potential financial conflict of interests related to this manuscript.

\section{Authors' Contributions}

Zhiyuan Zhao, Dongmei Miao, Kathleen Waugh, Iman Taki, and Fran Dong researched data and reviewed manuscript. Edwin Liu wrote/edited manuscript. Marian Rewers researched data and reviewed/edited manuscript. Yu Liu and Liping Yu researched data and wrote/edited manuscript. Zhiyuan Zhao and Dongmei Miao contributed equally to this work.

\section{Acknowledgments}

This research was supported by NIH Grants DK32083, DK32493, DK50979, and DK57516 and the JDRF Grant 2SRA-2015-51-Q-R.

\section{References}

[1] C. Dubé, A. Rostom, R. Sy et al., "The prevalence of celiac disease in average-risk and at-risk Western European populations: a systematic review," Gastroenterology, vol. 128, no. 4, pp. S57S67, 2005.

[2] A. Fasano, I. Berti, T. Gerarduzzi et al., "Prevalence of Celiac disease in at-risk and not-at-risk groups in the United States: a 
large multicenter study," Archives of Internal Medicine, vol. 163, no. 3, pp. 286-292, 2003.

[3] S. Lohi, K. Mustalahti, K. Kaukinen et al., "Increasing prevalence of coeliac disease over time," Alimentary Pharmacology and Therapeutics, vol. 26, no. 9, pp. 1217-1225, 2007.

[4] A. Myléus, A. Ivarsson, C. Webb et al., "Celiac disease revealed in 3\% of Swedish 12-year-olds born during an epidemic," Journal of Pediatric Gastroenterology and Nutrition, vol. 49, no. 2, pp. 170-176, 2009.

[5] C. Catassi, D. Kryszak, B. Bhatti et al., "Natural history of celiac disease autoimmunity in a USA cohort followed since 1974," Annals of Medicine, vol. 42, no. 7, pp. 530-538, 2010.

[6] S. Dydensborg, P. Toftedal, M. Biaggi, S. T. Lillevang, D. G. Hansen, and S. Husby, "Increasing prevalence of coeliac disease in Denmark: a linkage study combining national registries," Acta Paediatrica, vol. 101, no. 2, pp. 179-184, 2012.

[7] A. Rosén, O. Sandström, A. Carlsson et al., "Usefulness of symptoms to screen for celiac disease," Pediatrics, vol. 133, no. 2, pp. 211-218, 2014.

[8] M. F. Kagnoff, "AGA Institute medical position statement on the diagnosis and management of celiac disease," Gastroenterology, vol. 131, no. 6, pp. 1977-1980, 2006.

[9] J. L. Chiang, M. S. Kirkman, L. M. B. Laffel, and A. L. Peters, "Type 1 diabetes through the life span: a position statement of the American Diabetes Association," Diabetes Care, vol. 37, no. 7, pp. 2034-2054, 2014.

[10] S. Husby, S. Koletzko, I. R. Korponay-Szabó et al., "ESPGHAN Working Group on Coeliac Disease Diagnosis; ESPGHAN Gastroenterology Committee; European Society for Pediatric Gastroenterology, Hepatology, and Nutrition. European Society for Pediatric Gastroenterology, Hepatology, and Nutrition guidelines for the diagnosis of coeliac disease," Journal of Pediatric Gastroenterology and Nutrition, vol. 54, pp. 136-160, 2012.

[11] G. Adornetto, L. Fabiani, G. Volpe et al., "An electrochemical immunoassay for the screening of celiac disease in saliva samples," Analytical and Bioanalytical Chemistry, vol. 407, no. 23, pp. 7189-7196, 2015.

[12] M. Li, L. Yu, C. Tiberti et al., "A report on the international transglutaminase autoantibody workshop for celiac disease," American Journal of Gastroenterology, vol. 104, no. 1, pp. 154$163,2009$.

[13] M. Rewers, T. L. Bugawan, J. M. Norris et al., "Newborn screening for HLA markers associated with IDDM: diabetes autoimmunity study in the young (DAISY)," Diabetologia, vol. 39, no. 7, pp. 807-812, 1996.

[14] W. A. Hagopian, H. Erlich, Å. Lernmark et al., "The environmental determinants of diabetes in the young (TEDDY): genetic criteria and international diabetes risk screening of 421000 infants," Pediatric Diabetes, vol. 12, no. 8, pp. 733-743, 2011.

[15] L. Yu, D. Miao, L. Scrimgeour, K. Johnson, M. Rewers, and G. S. Eisenbarth, "Distinguishing persistent insulin autoantibodies with differential risk: nonradioactive bivalent proinsulin/insulin autoantibody assay," Diabetes, vol. 61, no. 1, pp. 179-186, 2012.

[16] L. Yu, F. Dong, D. Miao, A. R. Fouts, J. M. Wenzlau, and A. K. Steck, "Proinsulin/insulin autoantibodies measured with electrochemiluminescent assay are the earliest indicator of prediabetic islet autoimmunity," Diabetes Care, vol. 36, no. 8, pp. 2266-2270, 2013.
[17] D. Miao, K. M. Guyer, F. Dong et al., "GAD65 autoantibodies detected by electrochemiluminescence assay identify high risk for type 1 diabetes," Diabetes, vol. 62, no. 12, pp. 4174-4178, 2013.

[18] D. Miao, A. K. Steck, L. Zhang et al., "Electrochemiluminescence assays for insulin and glutamic acid decarboxylase autoantibodies improve prediction of type 1 diabetes risk," Diabetes Technology \& Therapeutics, vol. 17, no. 2, pp. 119-127, 2015.

[19] G. Oberhuber, "Histopathology of celiac disease," Biomedicine and Pharmacotherapy, vol. 54, no. 7, pp. 368-372, 2000.

[20] F. Bao, L. Yu, S. Babu et al., "One third of HLA DQ2 homozygous patients with type 1 diabetes express celiac disease-associated transglutaminase autoantibodies," Journal of Autoimmunity, vol. 13, no. 1, pp. 143-148, 1999.

[21] J. M. Norris, K. Barriga, E. J. Hoffenberg et al., "Risk of celiac disease autoimmunity and timing of gluten introduction in the diet of infants at increased risk of disease," Journal of the American Medical Association, vol. 293, no. 19, pp. 2343-2351, 2005.

[22] E. Liu, H.-S. Lee, C. A. Aronsson et al., "Risk of pediatric celiac disease according to HLA haplotype and country," New England Journal of Medicine, vol. 371, no. 1, pp. 42-49, 2014.

[23] E. Liu, M. Li, F. Bao et al., "Need for quantitative assessment of transglutaminase autoantibodies for celiac disease in screeningidentified children," Journal of Pediatrics, vol. 146, no. 4, pp. 494-499, 2005. 


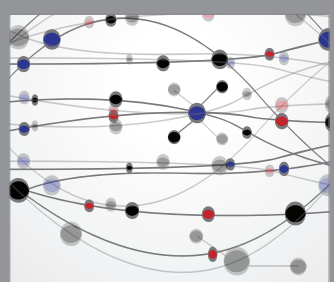

The Scientific World Journal
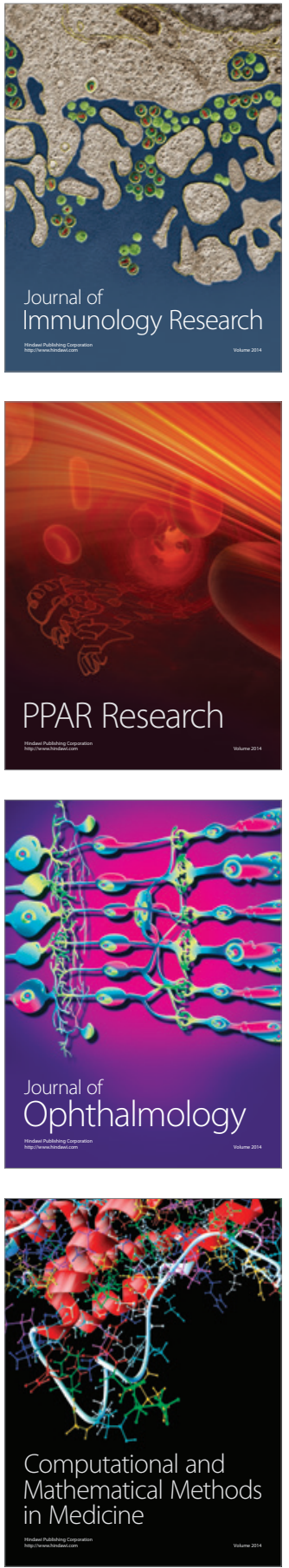

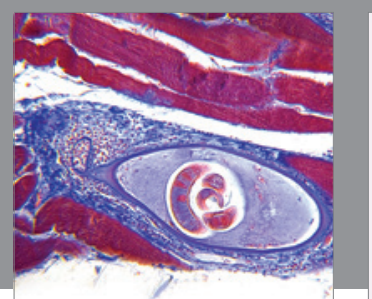

Gastroenterology Research and Practice
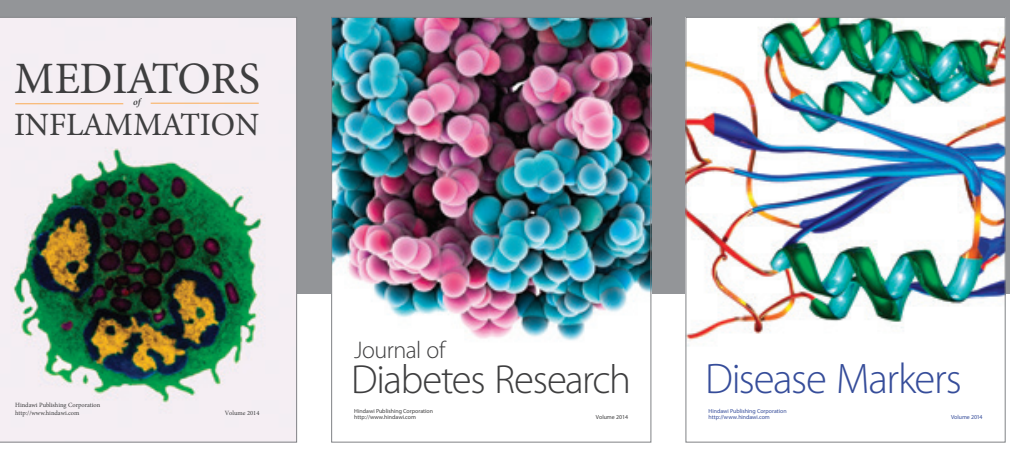

Disease Markers

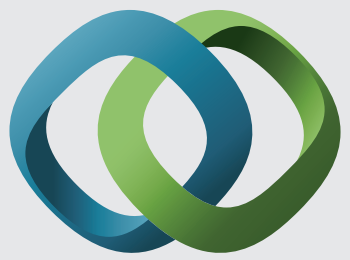

\section{Hindawi}

Submit your manuscripts at

https://www.hindawi.com
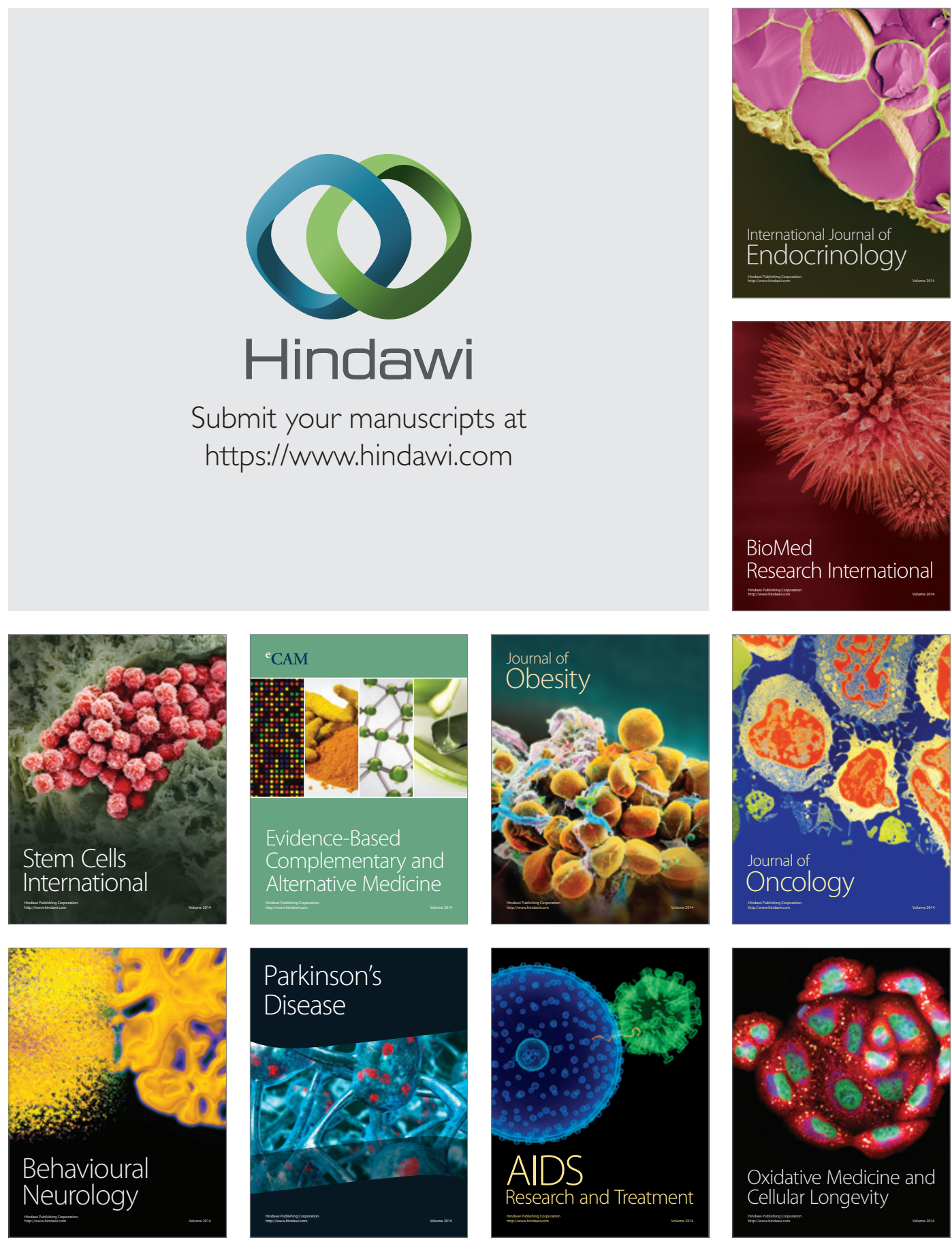